\title{
Excision of a part of the bile duct as an iatrogenic injury typical for laparoscopic cholecystectomy - characteristics, treatment and long-term results, based on own material
}

\author{
Sergiusz Durowicz, Ireneusz Kozicki, Adam Ciesielski, Wiesław Tarnowski \\ Department of General, Oncological and Digestive Tract Surgery, Centre of Postgraduate Medical Education, Warsaw, Poland
}

Videosurgery Miniinv 2020; 15 (1): 70-79

DOI: https://doi.org/10.5114/wiitm.2019.85806

\begin{abstract}
Introduction: Cholecystectomy is associated with the risk of bile duct injury (BDI). The nature of the injury in laparoscopic cholecystectomy (LC) cases seems to be more serious.

Aim: We present an analysis of long-term results of the treatment of patients who underwent operations at our department due to iatrogenic excision of a part of the bile duct (EPBD).

Material and methods: Out of all 120 patients treated for BDI in our department we selected a group of 40 with EPBD. In all cases the corrective operation was hepaticojejunostomy. The median follow-up time was 157 (56-249) months. We evaluated risk factors for EPBD during LC compared to open cholecystectomy (OC).

Results: Among bile duct injuries referred to our centre, EPBD occurred more frequently during LC (46.7\%) compared to OC (11\%), $p<0.001$. Injuries located in the hepatic hilum occurred more often in the case of LC (68.6\%) than OC (20\%), $p=0.056$. We did not find a difference in the frequency of EPBD between LC and OC groups depending on the presence of acute or chronic cholecystitis. The narrow common hepatic duct was reported more frequently in the LC (68.6\%) vs. OC (20\%) group, $p=0.056$. Satisfactory long-term reconstructive treatment results were observed in $36(90 \%)$ of 40 patients.

Conclusions: Excision of a part of the bile duct occurs more often during LC than OC. It is often located in the hepatic hilum. Presence of a narrow common hepatic duct is a risk factor for EPBD during LC. Large diameter hepaticojejunostomy is a reconstructive procedure that promises good long-term results.
\end{abstract}

Key words: laparoscopic cholecystectomy, long-term outcome, risk factors for excision of a part of bile duct, repair of major bile duct injury.

\section{Introduction}

Cholecystectomy has always been associated with the risk of bile duct injury (BDI). The risk has even increased since cholecystectomy performed by the laparoscopic method (laparoscopic cholecystectomy - LC) has become the accepted standard for the treatment of cholelithiasis. Over the years, the results of many studies have suggested that patients are more exposed to BDI when they are op- erated on with a laparoscopic technique, compared to the open method (open cholecystectomy - OC). However, recent results indicate that this principle has changed [1, 2]. With the development of laparoscopic techniques, the rates of injuries in both groups have gradually got closer to each other. However, the nature of the injury seems to be different and it is more serious in cases of LC. In the literature considering $\mathrm{BDI}$, the possible types of injury were

\section{Address for correspondence}

Sergiusz Durowicz MD, PhD, Department of General, Oncological and Digestive Tract Surgery, Centre of Postgraduate Medical Education, 231 Czerniakowska St, 00-416 Warsaw, Poland, e-mail: sdurowicz@wp.pl 
classified in detail, factors that increase its risk were analysed, diagnostic procedures were developed and treatment methods were suggested, with the presentation of their results [3-7]. However, there is a lack of studies presenting long-term results of reconstructive surgery in a selected group of patients with a major type of bile duct injury, involving excision of a part of the bile duct (EPBD) following LC as well as OC. Such a study is at present difficult to carry out, because the number of patients operated on by the OC method has significantly decreased. In addition, among the smaller group of patients undergoing OC surgery, it is difficult to collect subjects with an excision of a segment of the bile duct, because this kind of injury is considered more characteristic of the laparoscopic method [8-12].

\section{Aim}

The aim of the study is to present an analysis of long-term results of the treatment of patients who underwent operations at our department due to severe injuries of the EPBD type following $L C$ as well as OC.

\section{Material and methods}

One hundred and twenty patients with diagnosed BDI were referred to our clinic, which is a reference unit, from other surgical departments for corrective operations between 1996 and 2013. In 75 patients, the injury occurred during LC and in 45 during OC. The majority of the discussed patients were admitted after a post-operative revision performed in a primary department, and some of them were admitted directly after the injury. The most common type of intervention during post-operative revision was drainage of the liver hilum area with biliary decompression. However, there were also patients who had undergone ineffective attempts of repair surgery.

During the last 5 years, we continued to treat surgically patients with BDI. These patients are not included in this study due to the too short period of postoperative follow-up.

Out of all 120 discussed patients with BDI, we distinguished and analysed a group of 40 patients with a more severe form of injury in the form of excision of a part of the bile duct. We assigned patients to this group on the basis of the description of the operation during which the injury occurred, as well as the image that was found during the reconstruction performed by us. The diagnosis was also confirmed before reconstruction with imaging, almost in each case with endoscopic retrograde cholangiopancreatography (ERCP) and in the last 8-year period with magnetic resonance of the bile ducts (MRCP). The gap between the proximal stump or stumps and the distal stump of the bile ducts exceeded $4 \mathrm{~cm}$. In the group of patients with EPBD we compiled data on age, sex, the level of injury according to the Strasberg classification [3], coexistence of vascular injury, time from the injury to its diagnosis, the type of pre-surgery performed at the department where the patient was initially operated, the type of corrective operation performed at our department and its long-term results.

In all cases of suspected vascular injury (clipping of the right hepatic artery (RHA)), the diagnosis was made at the surgical ward in which the injury occurred and was based solely on the intraoperative assessment.

We also determined the presence of additional factors that may have made it difficult to assess the anatomical topography properly during the primary surgery. Such factors include the presence of: 1) a narrow, up to $3 \mathrm{~mm}$ in diameter, common hepatic duct (CHD), 2) acute inflammation of the gallbladder (acute cholecystitis - AC), 3) a fibrotic, shrunken gallbladder with deposits, as a result of the course of chronic inflammation (chronic cholecystitis - CC) and 4) Mirizzi syndrome.

In all patients with EPBD, the corrective operation, which was performed at our department, was anastomosis of the common hepatic duct with the separated loop of jejunum, i.e. hepaticojejunostomy $(\mathrm{HJ})$.

After location of the site of injury, we isolated the confluence of the right and left hepatic ducts (E2 and E3) or separately left and right hepatic ducts (E4). It was crucial for us to preserve confluence, because it is much easier to apply the anastomosis to a single duct. Moreover, confluence injury can disrupt the hilar vascular shunt, which makes collateral blood flow from the left hepatic artery impossible in the case of an RHA injury. In cases of difficulties to achieve sufficiently wide anastomosis, we used the Hepp-Couinaud technique, i.e. a longitudinal incision of the anterior wall of the left hepatic duct $[11,13]$. We tried to make a wide incision on the left duct, about 15 to $20 \mathrm{~mm}$, and obtain good communication between the right and left sides. The leftward 
extent of the incision should stop in the place where the artery to segment 4 crosses the duct anteriorly. When, in E4 type injury, a separate right and left duct were found, we always endeavoured to approximate them and combine them together. Sometimes, for this purpose we had to destroy the liver parenchyma between them. Such a procedure has been significantly facilitated since the appearance of electrosurgical devices. This made it possible to continue with further procedures as if we were dealing with a single duct. Next, in each of the above - described cases, a jejunal Roux limb was brought up to the liver hilum and the ductal stump was anastomosed in end-toside configuration. The length of the Roux limb was between 50 and $60 \mathrm{~cm}$, and the distance from the ligament of Treitz was around $20-30 \mathrm{~cm}$.

In 1 patient, because of a small, weak bile duct, we left a trans-anastomotic 6 Fr stent. The catheter was passed through the lumen of the jejunal limb for about $20 \mathrm{~cm}$ and brought outside. We removed the drain three weeks after the operation, after performing contrast cholangiography.

During the reconstruction, we paid a lot of attention to the diameter of the anastomosis that we wanted to achieve. For systematization, the width of the anastomoses has been divided into the following categories: A - wider than $15 \mathrm{~mm}$; B - from 10 to $15 \mathrm{~mm}$; $\mathrm{C}$ - from 5 to $10 \mathrm{~mm}$; $\mathrm{D}$ - narrower than $5 \mathrm{~mm}$.

Patients were called in and subjected to follow-up examinations 12 months after reconstructive surgery. These examinations included the clinical evaluation of the patient, the determination of biochemical markers of cholestasis in the blood serum, such as the levels of bilirubin, alkaline phosphatase, gamma-glutamyltransferase, and alanine and aspartate transaminase. Imaging examinations were also performed: in each case abdominal ultrasound,

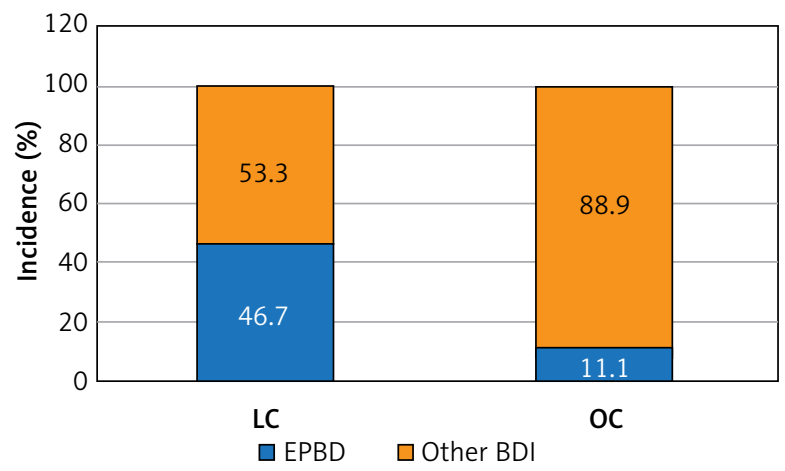

Figure 1. Frequency of EPBD during $L C$ and $O C$ in some patients MRCP. After the next 12 months, full follow-up examinations were performed only in patients with biochemical suspicion of cholestasis. In addition, patients were asked to contact our department whenever they had any worrying symptoms that might have indicated cholangitis, such as increased body temperature, chills, and icterus.

\section{Statistical analysis}

Descriptive statistics are presented as the number, mean, standard deviation, minimum and maximum for continuous variables and as the number and percentage for categorical variables. The comparison between OC and LC groups was assessed using the Mann-Whitney test for continuous variables and the $\chi^{2}$ or Fisher's exact test for categorical variables. The analysis was performed using SPSS 25.0 statistical software.

\section{Results}

We diagnosed the injury of EPBD type in 35 out of 75 patients with BDI that occurred during LC $(46.7 \%)$ and in 5 out of 45 patients with BDI that occurred during OC (11\%). By means of the $\chi^{2}$ test of independence, an analysis of the rate of occurrence of EPBD depending on the type of surgery was performed. The analysis showed that the difference was statistically significant, $\chi^{2}(1)=14.44 ; p<0.001$. In patients after LC, EPBD occurs significantly more frequently than in patients after OC. Figure 1 illustrates the results.

In the course of the study, only a group of 40 patients diagnosed with EPBD was subjected to analysis. This group consisted of 34 women and 6 men, aged 24 to 75, median value 51 years. The ratio of women to men among patients with OC and LC was similar $(p>0.999)$. The characteristics of the analysed sample are presented in Table I.

Injuries located at the level of the confluence connecting the hepatic ducts in the hepatic hilum, type E3 or E4 according to the Strasberg classification, were found respectively in 19 and 6, in summary in 25 patients, and distal from the confluence, type E2, in 15 patients. Injuries located in the hepatic hilum, type E3 and E4 together, occurred more often in the case of LC (68.6\%) than OC (20\%), with borderline significance, $p=0.056$.

Acute cholecystitis was observed in 15 (53.6\%) patients during cholecystectomy, during which the 
Table I. Characteristics of the studied group of patients with EPBD

\begin{tabular}{|lccc|}
\hline Parameter & LC & OC & Summary \\
\hline Patients, $n(\%)$ & $35(87.5)$ & $5(12.5)$ & $40(100.0)$ \\
\hline Age, median (range) [years] & $50(24-75)$ & $67(28-75)$ & $51(24-75)$ \\
\hline \begin{tabular}{l} 
Gender, $n(\%):$ \\
\hline Female
\end{tabular} & $30(85.7)$ & & \\
\hline Male & $5(14.3)$ & $1(80.0)$ & $34(100.0)$ \\
\hline
\end{tabular}

injury occurred, fibrosis of the gallbladder and its contraction with deposits as a result of chronic inflammation was observed in 10 (35.7\%) patients, and Mirizzi syndrome was observed in $3(10.7 \%)$ patients. We did not find a significant difference in the frequency of EPBD injuries between LC and OC groups depending on the presence of inflammation at the operated site.

A narrow $\mathrm{CHD}$, up to $3 \mathrm{~mm}$ in diameter, was reported in 25 (62.5\%) patients - in $68.6 \%$ of patients operated on by the laparoscopic method and in $20 \%$ operated on by the open method. Statistical analysis showed only a borderline significant difference between these two groups of patients $(p=0.056)$.

Bile duct injury was accompanied by a vascular injury in the form of a closure of the RHA with a surgical clip in 8 (20\%) patients. All these cases concerned patients who were operated on laparoscopically. However, the comparison of the incidence of vascular injury between patients after OC and LC, using Fisher's exact test, showed no statistical difference $(p=0.56)$. Reconstructive operations in patients with vascular injury were performed at a distant time, from 6 weeks to 20 months (median: 4.25 months) following injury.

The excision of the bile ducts was not recognized immediately in 25 (62.5\%) patients. In these patients, the delay time to surgical intervention took 2-45 days, on average 10 days. The surgical intervention after the diagnosis of bile duct injury was performed in the primary department in 31 of 40 patients. The following procedures were performed: external drainage in 21 cases, end-to-end ductal anastomosis in 2 cases, and $\mathrm{HJ}$ in 8 cases.

We did not find significant differences between LC and OC groups in terms of the frequency of cases in which the injury was not noted during cholecystectomy, the time passed before its diagnosis and the type of operative intervention undertaken at the department where the patient was initially operated on ( $p>0.999, p=0.23, p=0.106$, respectively).

We performed $\mathrm{HJ}$ as a reconstructive operation in all cases in the group of discussed patients. The time from injury to reconstruction was from 7 days to 20 months (median: 2 months). In 22 cases we used the Hepp-Couinaud technique. In 4 out of 6 patients with E4 type injury according to the Strasberg classification we combined the circumferences of both separately found hepatic ducts before the anastomosis.

We assigned the width of the anastomosis, in accordance with the definition we adopted, to the following categories: A - in 26 cases, B - in 12 cases, $C$ - in 1 case and D - in 1 case. The $L C$ and OC groups did not differ significantly in terms of the width of the biliary-intestinal anastomosis that we achieved.

The aggregate results of the comparative analyses of patients after LC and OC are presented in Table II.

The median follow-up time after a reconstructive operation was 157 months (i.e. over 13 years), ranging from 56 to 249 months.

We did not find any results that could have indicated the presence of cholestasis or damage of the liver tissue in follow-up laboratory tests, which were performed 12 months after a reconstructive operation. In total, in the LC and OC groups, the mean serum level of bilirubin was $0.6 \pm 0.2 \mathrm{mg} / \mathrm{dl}$, that of alkaline phosphatase was $78 . \pm 23.3 \mathrm{U} / \mathrm{l}$, that of gamma-glutamyltransferase was $31.6 \pm 9.5 \mathrm{U} / \mathrm{l}$, that of alanine transaminase was $32.6 \pm 10.7 \mathrm{U} / \mathrm{l}$, and that of aspartate transaminase was $27.4 \pm 5.0 \mathrm{U} / \mathrm{l}$. All results were within the reference range of our analytical laboratory. The results, which are separated into LC and OC groups, are presented in Table III.

On the basis of anamnesis and physical examination of patients, we assessed the quality of their life as good. The degree of fitness according to the 
Table II. Comparison of patients with EPBD following LC and OC

\begin{tabular}{|c|c|c|c|c|}
\hline Variable & LC & OC & Summary & $P$-value \\
\hline \multicolumn{5}{|l|}{$\begin{array}{l}\text { Level of injury according to the Strasberg } \\
\text { classification }\end{array}$} \\
\hline E2 (distal) & $11(31.4 \%)$ & $4(80.0 \%)$ & $15(37.5 \%)$ & \multirow[t]{2}{*}{0.056} \\
\hline $\mathrm{E} 3$ + E4 (hilum) & $24(68.6 \%)$ & $1(20.0 \%)$ & $25(62.5 \%)$ & \\
\hline E3 & $18(75.0 \%)$ & $1(100.0 \%)$ & $19(76.0 \%)$ & \multirow[t]{2}{*}{$>0.999$} \\
\hline E4 & $6(25.05)$ & $0(0 \%)$ & $6(24.0 \%)$ & \\
\hline Vascular injury (right hepatic artery) & $8(22,9 \%)$ & $0(0 \%)$ & $8(20.0 \%)$ & 0.563 \\
\hline Narrow bile duct ( $\leq 3 \mathrm{~mm})$ & $24(68,6 \%)$ & $1(25 \%)$ & $25(62.5 \%)$ & 0.056 \\
\hline \multicolumn{5}{|l|}{ Inflammatory conditions } \\
\hline Acute cholecystitis & $12(52.5 \%)$ & $3(20.0 \%)$ & $15(53.6 \%)$ & \multirow[t]{3}{*}{$>0.999$} \\
\hline Chronic cholecystitis & $8(34.8 \%)$ & $2(20.0 \%)$ & $10(35.7 \%)$ & \\
\hline Mirizzi syndrome & $3(13.0 \%)$ & $0(0 \%)$ & $3(10.7 \%)$ & \\
\hline BDI intraoperatively unrecognized & $22(62.9 \%)$ & $3(60 \%)$ & $25(62.5 \%)$ & $>0.999$ \\
\hline Diagnosis delayed, average \pm SD [days] & $9.27 \pm 10.78$ & $11.00 \pm 6.08$ & $9.48 \pm 10.25$ & 0.238 \\
\hline \multicolumn{5}{|l|}{ Surgery performed before referring patients } \\
\hline External drainage & $20(57.1 \%)$ & $1(20.0 \%)$ & $21(52.0 \%)$ & \multirow[t]{3}{*}{0.106} \\
\hline $\mathrm{H}-\mathrm{J}$ & $7(20.0 \%)$ & $1(20.0 \%)$ & $8(20.0 \%)$ & \\
\hline End-to-end ductal anastomosis & $1(2.9 \%)$ & $1(20.0 \%)$ & $2(5.0 \%)$ & \\
\hline \multicolumn{5}{|l|}{$\begin{array}{l}\text { Surgery performed at our department - } \\
\text { anastomotic diameter }\end{array}$} \\
\hline $\mathrm{A}(>15 \mathrm{~mm})$ & $21(60.0 \%)$ & $5(100.0 \%)$ & $26(65.0 \%)$ & \multirow[t]{4}{*}{0.358} \\
\hline $\mathrm{B}(10-15 \mathrm{~mm})$ & $12(34.2 \%)$ & $0(0 \%)$ & $12(30.0 \%)$ & \\
\hline $\mathrm{C}(5-10 \mathrm{~mm})$ & $1(2.9 \%)$ & $0(0 \%)$ & $1(2.5 \%)$ & \\
\hline $\mathrm{D}(<5 \mathrm{~mm})$ & $1(2.9 \%)$ & $0(0 \%)$ & $1(2.5)$ & \\
\hline
\end{tabular}

$p$-significance level.

Table III. Laboratory tests performed 12 months following reconstructive surgery

\begin{tabular}{|lcc|}
\hline Blood serum level & LC group & OC group \\
\hline Total bilirubin [mg/dl] & $0.6(0.2)$ & $0.9(0.1)$ \\
\hline Alanine aminotransferase [Ul] & $34.1(11.4)$ & $33.0(14.9)$ \\
\hline Aspartate aminotransferase [U/l] & $28.3(5.2)$ & $24.6(0.9)$ \\
\hline Alkaline phosphatase [U/l] & $75.5(23.3)$ & $88.0(19.8)$ \\
\hline$\gamma$-Glutamyltransferase [U/l] & $31.9(8.6)$ & $32.6(14.1)$ \\
\hline
\end{tabular}

All values were within our analytical laboratory reference range.

Zubrod-ECOG scale was defined as "0", meaning "normal fitness, ability to perform everyday activities on one's own". In the late follow-up period we considered objective assessment of the quality of life due to the disease - bile duct injury - as impossible due to the significant influence on the self-as- sessment of ailments resulting from other diseases, often in elderly patients.

During the follow-up period in 4 patients ( 2 women and 2 men), we found a complication in the form of biliary-intestinal anastomosis insufficiency. The complication occurred at a distant time from the surgical treatment - from 28 to 96 months (median: 60 months). In the first case, in the MRCP imaging examination, we found a significant narrowing of the bile duct. The patient was successfully re-operated. In the second case, there occurred a complication of pulmonary embolism and death of the patient before the planned surgery. We did not find any significant stenosis of the bile duct in the 2 other cases and the symptoms of cholangitis were effectively treated with conservative methods. Of the remaining patients we observed, 2 died from causes independent of biliary tract disease, without 
any signs of cholestasis. Cardiovascular insufficiency in the elderly was the cause of death in both cases.

We were forced to interrupt the observations in two patients due to the lack of cooperation on their part, after 12 and 36 months. One of the patients suffered from a mental illness, the other one from multiple sclerosis. However, it should be noted that in both cases the anastomosis that was made during the reconstruction of the bile duct was wide and promising for sustained success. In addition, until the last moment of contact with the patients, we did not record any signs of cholestasis.

The remaining patients did not present clinical, laboratory or imaging indicators of insufficiency of biliary-intestinal anastomosis. Thus, satisfactory long-term results concern 36 (90\%) of 40 patients.

Results regarding late complications and mortality of patients are presented in Table IV.

\section{Discussion}

Topics concerning bile duct injuries are still of great scientific interest. The risk of BDI in the era of open cholecystectomy was reported as $0.1-0.2 \%$. The risk increased almost tenfold shortly after the introduction of laparoscopic surgery. Over the years, large US studies concerning BDI that were based on statistics taken from the national database showed a gradual decrease in the frequency of BDI after LC. Currently, laparoscopic cholecystectomy in the United States and Canada is no longer associated with a greater risk of BDI compared to open cholecystectomy. Mangieri et al. identified the risk of $\mathrm{BDI}$ requiring reconstructive surgical intervention after LC at 0.19\% [1].

Authors from the United Kingdom, the United States and Australia, in a systematic review and analysis of aggregate data of 505,292 patients gathered from around the world, presented the risk of BDI after LC at the level of 0.32-0.52\%. However, unlike in the work previously cited, the authors collectively qualified as BDI serious injuries that required reconstructive surgical intervention and, more often, injuries in which only a leak of bile was observed and which were treated endoscopically. The incidence of serious injuries was $0.28 \%$, and that of only bile leak was $0.46 \%$ [2].

According to above-mentioned reports, the conversion rate, even in inflammation when there is difficulty in tissue preparation or if gallbladder perforation occurs, is remarkably low. Such management
Table IV. Late complications and mortality following reconstructive surgery

\begin{tabular}{|lcc|}
\hline Variable & Number & Percentage \\
\hline \begin{tabular}{l} 
Late complications: \\
\hline Anastomotic stricture
\end{tabular} & 2 & 5 \\
\hline Cholangitis & 4 & 10 \\
\hline Mortality: & & \\
\hline $\begin{array}{l}\text { related to bile duct injury } \\
\text { not related to bile duct injury }\end{array}$ & 1 & 2.5 \\
\hline
\end{tabular}

to avoid the conversion seems to be correct because when a cholecystectomy requires conversion from a laparoscopic to an open approach the BDI increases by as much as a hundredfold. Sahbaz et al. stated that all types of gallbladder perforation, without making a distinction between acute, subacute or chronic cholecystitis, can be safely treated without conversion, by the laparoscopic approach [14].

The frequency of injury in LC and OC groups became closer to each other; however, the nature of the injury and the type of patients that are affected seem to vary. People who are injured are often young, professionally active, and the injury unexpectedly does not allow a quick return to full activity. Moreover, these patients do not always return to full health after the period of surgical treatment and reconvalescence [3-7, 12, 15].

The EPBD has been recognized for years as a "classic" injury for cholecystectomy performed using the laparoscopic method. The danger usually exists when a narrow common hepatic duct (CHD) is hidden behind the neck of the gallbladder. It is easy to confuse the common bile duct with the cystic duct. A hidden section of the CHD may then be cut out together with the gallbladder [3-7, 16, 17].

This pattern is confirmed in our research. We diagnosed EPBD type injury in almost $47 \%$ of patients with BDI which happened during LC and only in $11 \%$ of patients with BDI which happened during OC. This difference was statistically significant. One needs to be aware of the fact that the frequency of EPBD in our material, proportionally in both the LC and OC groups, may be overstated. Usually selected patients were referred to our department with BDI that was harder to treat.

Our observations included in this study date back to 1996 . We can observe that over time, the arsenal of diagnostic and operational capabilities has 
increased. For example, in the initial period, imaging diagnostics in the discussed patients was based only on ERCP, and later we gradually gained access to MRCP. Today, the most useful imaging methods include ERCP, MRCP, and computed tomography (CT) with angiography. Each of these methods has its various advantages and limitations, and each compliment others. With ERCP, the biliary system is evaluated only distally to the level of duct transection or ligation. However, it allows simultaneous therapeutic interventions such as the placement of biliary stents and drainage catheters, which are standard for treating smaller injuries. MRCP is non-invasive, does not require the use of a contrast medium, and provides excellent delineation of the biliary anatomy proximal and distal to the level of injury. MRCP facilitates the identification of fluid collections and, if performed with use of an intravenous contrast medium, arterial injuries. The accuracy of contrast-enhanced MRCP performed with a hepatocyte-selective contrast agent for the detection of bile leakage is close to $100 \%$, and the exact location of leakage can be determined in approximately $79-85 \%$ of such examinations [18] And finally, CT angiography is indicated when vascular injury, especially in the early postoperative period, is suspected. It can among other things precisely depict occlusion or other signs of arterial injury such as narrowing, intraluminal thrombus or pseudoaneurysm. Very rarely, vascular injuries lead in the early postoperative period (within a few weeks) to liver parenchymal necrosis and sepsis. Such changes, in turn, may lead to the need for urgent hepatectomy [19]. Fortunately, we did not deal with such cases.

The time of repair surgery from the occurrence of the injury was variable and ranged from 7 days to 20 months. Of the 40 patients with EPBD, we decided to perform reconstructive surgery shortly after BDI only in 4 of them. We did not suspect a vascular injury in any of these patients. They were referred to us faster than most other patients, and immediate revision surgery was necessary due to insufficient drainage of the hepatic hilum and the need of bile decompression. During the inspection, we found favourable conditions for simultaneous reconstructive surgery, that is, sufficient diameter of the bile duct stump and its good blood supply. In most other cases, during similar surgical revisions, we made the decision to delay the reconstruction.

The analysis of the group of discussed patients shows that during performance of $L C$ the risk factors for EPBD include the occurrence of a narrow CHD. There is agreement in the existing literature that the small diameter of the CHD and acute inflammation of the gallbladder are risk factors for BDI after each type of surgery [3, 4, 11, 17, 20-24], whereas only one study mentions the presence of a small, inflammation-induced fibrotic, contracted gallbladder with deposits as a risk factor for BDI [25]. In our material, the occurrence of a small, inflammation-induced fibrotic gallbladder predisposes to BDI also during OC (this took place in 3 out of 5 cases).

In the literature, the presence of cholecystitis increases the risk of a BDI. Therefore, especially in the presence of severe pericholecystic inflammation making it difficult to identify anatomical structures, it is recommended to refrain from immediate cholecystectomy. Beside conservative treatment, the minimally invasive methods of external bile drainage seem to be of special importance [26].

It is widely accepted that a narrow CHD occurs as a risk factor for injury mainly in young, slim women. Our analysis confirms this demographic trend, but there were also older people and men among those with an injury and a narrow CHD. We found a CHD of a diameter of $3 \mathrm{~mm}$ or less in 27 women and 3 men. The median age of patients with a narrow CHD was 46 years with the range of 28-73 years. Five of them exceeded 60 years of age.

EPBD was correctly diagnosed during LC operations in $37.5 \%$ of cases. In cases of missing diagnosis, the mean delay time for diagnosis was on average 10 days. In the majority of studies concerning $\mathrm{BDI}$, intraoperative diagnosis of injuries occurring during LC was reported less frequently, in approximately $25 \%$ to $33 \%$ of patients. However, the time delay in diagnosing the injury in most studies was similar to that recorded by us [3-7, 22]. Interestingly, the intraoperative recognition of BDI that was caused during $\mathrm{OC}$ according to the literature is much higher, more than 50\% [3-7]. In our material, we did not find a significant difference in the frequency of intraoperative recognition of injury between $\mathrm{LC}$ and OC groups. The delay time to make the diagnosis was also similar in both groups.

We did not find any statistical association between the number of late complications after our repair operations and the time of diagnosis of injury (intraoperative vs postoperative) or the type of procedure performed in the primary centre, because our research material was too small. However, in our 
opinion, when a lesion is identified and the surgeon performing the operation does not have adequate experience in bile duct surgery, the procedure should be limited to proper drainage and if necessary bile duct decompression. Subsequently, the patient should be referred to a tertiary centre. This practice, without making an inadequate attempt to repair the damage, will significantly facilitate subsequent reconstructive surgery in the reference centre.

According to the literature, extensive bile duct injuries located close to the liver hilum are more common during LC than OC [3, 9-11, 22, 27-32]. This is also confirmed by our observations: we recorded the injury in the liver hilum, grade E3/E4 according to the Strasberg classification, in $24(68.6 \%)$ patients after LC, and only in one patient (20\%) after OC. However, because of too few patients to compare, the statistical analysis only showed a borderline significant difference between these two groups $(p=0.056)$. The fact that BDI during $\mathrm{LC}$ occurs more often close to the hepatic hilum is probably due to altered anatomical conditions resulting from the filling of the peritoneal cavity with gas. The shift of organs causes the exposure of the area in which the proximal section of the $\mathrm{CHD}$ is present.

$\mathrm{BDI}$ was associated with hepatic artery injury in $8(20 \%)$ patients. All cases of such a combined injury were observed in laparoscopic patients in our material. Our results are in accordance with the observations in the literature [12, 28]. However, again, the small number of patients in both groups did not allow us to demonstrate that this association was statistically significant.

All 8 discussed patients with occlusion of the RHA were referred to our clinic within a long time after the injury. Due to their good general condition and the necessity of reconstructive surgery in each case, we decided to assess the blood supply of the biliary stumps intraoperatively. Only one of these patients revealed later insufficiency of biliary-intestinal anastomosis. He was referred to our unit late, 20 months after injury, following an unsuccessful attempt of $\mathrm{HJ}$ and was seriously burdened with additional diseases. He died as a result of pulmonary embolism before the planned next surgery. In our opinion, we cannot state with certainty that his anastomotic insufficiency was directly related to vascular injury. In the remaining seven cases, the result of our reconstructive surgery is satisfactory. We did not observe the need to perform arterial imag- ing in these patients. According to experts' opinion, routine arteriography in patients with a biliary injury is recommended only if early repair is considered, because then the bile duct is often ischaemic and failures of reconstructions are more common [12]. There is still disagreement whether RHA injury coexisting with BDI has an influence on late anastomotic stricture formation. Some researchers claim that it worsens the late outcome, but our observations are consistent with those who have a different opinion. Tzovaras and Dervenis in a review of the literature found that the disruption of the hepatic arterial flow is usually well tolerated. There is no strong evidence to support a negative impact of a concomitant vascular injury on long-term anastomotic stricture formation. There is strong evidence that concomitant vascular injuries do not have any impact on mortality after biliary reconstruction [33]. The collateral circulation after the occlusion of the RHA appears from the unaffected side. Although it is already present after $10 \mathrm{~h}$, and arterial flows are approaching normal values after about 4 days, we prefer to delay the reconstruction of the bile ducts by at least 6 weeks. Similar timing of the biliary repair is recommended by world experts in bile duct surgery [12]. Furthermore, according to our observations, after waiting a few weeks, the walls of the bile ducts become thicker, stronger and easier to combine with the separated intestinal loop.

Data available in the literature suggest that a type of reconstructive surgery which may promise permanent success in patients with EPBD is anastomosis of the hepatic duct with an isolated jejunal loop [4-7]. It is very important to make an anastomosis with a sufficiently wide diameter to ensure adequate free flow of bile. The small calibre of the bile duct is a serious difficulty in the implementation of an intestinal anastomosis. The diameter of this anastomosis can then be increased with E2/E3 type injuries by using the Hepp-Couinaud technique [11, $13,31]$. An important additional advantage of this technique is that it allows the formation of an anastomosis on a well blood-supplied hepatic duct, even in a complex injury of the bile duct and the RHA [13]. According to our experience, the aforementioned procedure allows the creation of an anastomosis of a diameter of more than $15 \mathrm{~mm}$, even in the case of an injury that occurs close to the hepatic hilum. We observed that unsatisfactory results of our reconstructive procedures may result not only from 
the narrow diameter of the bile ducts, but also from the inability to perform the Hepp-Couinaud technique. This is the case, inter alia, when we deal with the narrowing of intrahepatic bile ducts due to the fibrotic process or the anatomically unfavourable course of the left hepatic duct, which is directed into the liver parenchyma.

In a patient in whom we left a trans-anastomotic $6 \mathrm{Fr}$ stent, the long-term result of treatment was good. However, it should be noted that in other similar cases, but treated by us without stenting, the results were comparable. In the literature, some authors routinely leave a stent in the bile duct during $\mathrm{HJ}$. They claim it improves the patency of the anastomosis in the early postoperative period and lowers the probability of its postoperative stricture [34]. Such practice also allows post-operative cholangiography in order to control anastomosis. On the other hand, other authors claim that the routine use of a biliary stent is not justified before or after surgery [35].

The long-term post-operative results achieved by us are good, as $90 \%$ of patients did not present symptoms.

We did not find any results that could have indicated the presence of cholestasis or damage of the liver tissue in follow-up laboratory tests, which were performed 12 months after a reconstructive operation. Interestingly, patients who developed complications in the following months also had normal results of the tests.

We observed late disease symptoms resulting from bile duct narrowing or symptoms of cholangitis without significant narrowing in diagnostic imaging examinations in 4 patients on whom we operated. These complications led to re-operation only in 1 case. In the second case there was a complication in the form of pulmonary embolism and death of the patient before the planned surgery. In the other 2 cases, patients with recurrent symptoms of cholangitis, many years after the corrective operation, remain under our observation. In the literature it is reported that inflammation of the bile ducts, as a complication after hepaticojejunostomy, is usually caused by narrowing of the anastomosis. We may be dealing with a small number of cases with the inflammatory conditions of discrete clinical symptoms, which are described by patients as mild flulike symptoms. These symptoms often occur without narrowing of the bile ducts and are attributed to the recurrence of gastro-intestinal contents to the biliary tree. This syndrome in the literature is referred to as sump syndrome. This syndrome occurs less commonly when using the appropriate length of the Roux loop. However, the lengthening of the loop to $70 \mathrm{~cm}$ or more does not always solve the problem, and then the only available therapeutic method is the chronic, rotational use of antibiotics [36].

\section{Conclusions}

Excision of a part of the bile duct is an injury that occurs more often during LC than OC. Moreover, this injury then has a more severe form: it is frequently located in the hepatic hilum, above the convergence of the hepatic ducts.

Risk factors for the occurrence of EPBD during LC include the presence of a narrow common hepatic duct.

Our experience, combined with a long period of observation of the patients treated by us, allows us to conclude that the $\mathrm{HJ}$ performed at the reference centre is a reconstructive procedure that promises good long-term results. The condition of therapeutic success is the creation of an anastomosis of a wide diameter. In cases in which there are difficulties related to this, good results can be obtained by using the Hepp-Couinaud technique.

\section{Conflict of interest}

The authors declare no conflict of interest.

\section{References}

1. Mangieri CW, Hendren BP, Strode MA, et al. Bile duct injuries (BDI) in the advanced laparoscopic cholecystectomy era. Surg Endosc 2019; 33: 724-30.

2. Pucher PH, Brunt LM, Davies N, et al. Outcome trends and safety measures after 30 years of laparoscopic cholecystectomy: a systematic review and pooled data analysis. Surg Endosc 2018; 32: 2175-83.

3. Strasberg SM, Hertl M, Soper NJ. An analysis of the problem of biliary injury during laparoscopic cholecystectomy. J Am Coll Surg 1995; 180: 101-25.

4. Connor S, Garden OJ. Bile duct injury in the era of laparoscopic cholecystectomy. Br J Surg 2006; 93: 158-68.

5. Kozicki I, Bielecki K. Jatrogenne urazy dróg żółciowych powstałe podczas cholecystektomii laparoskopowej (latrogenic injuries of bile ducts resulting from laparoscopic cholecystectomy). Post Sci Med 2006; 19: 36-40.

6. Stewart L. Iatrogenic biliary injuries. Identification, classification, and management. Surg Clin N Am 2014; 94: 297-310.

7. Eikermann M, Siegel R, Broedres I, et al. Prevention and treatment of bile duct injuries during laparoscopic cholecystectomy: 
the clinical practice guidelines of the European Association for Endoscopic Surgery (EAES). Surg Endosc 2012; 26: 3003-39.

8. Davidoff AM, Pappas TN, Murray EA, et al. Mechanisms of major biliary injury during laparoscopic cholecystectomy. Ann Surg 1992; 215: 196-202.

9. Branum G, Smitt C, Baillie J, et al. Management of major biliary complications after laparoscopic cholecystectomy. Ann Surg 1993; 217: 532-41.

10. Krawczyk M, Patkowski W. Taktyka postępowania w jatrogennym uszkodzeniu dróg żółciowych (Tactics for the management of iatrogenic bile duct injury). Pol Przeg Chir 2001; 73: 4-16.

11. Kozicki I, Bielecki K, Pertkiewicz J. Jatrogenne urazy dróg żółciowych podczas cholecystektomii laparoskopowych. Analiza problemu na podstawie leczenia 25 przypadków (latrogenic injuries of bile ducts during laparoscopic cholecystectomy. The analysis of a problem based on the treatment of 25 cases). Wideochirurgia 2005; 10: 13-6.

12. Strasberg SM, Helton WS. An analytical review of vasculobiliary injury in laparoscopic and open cholecystectomy. HPB (Oxford) 2011; 13: 1-14.

13. Murr MM, Gigot JF, Nagorney DM, et al. Long-term results of biliary reconstruction after laparoscopic bile ducts injuries. Arch Surg 1999; 134: 604-10.

14. Sahbaz NA, Peker KD, Kabuli HA, et al. Single center experience in laparoscopic treatment of gallbladder perforation. Videosurgery Miniinv 2017; 12: 372-7.

15. Way LW, Stewart L, Gantert W, et al. Causes and prevention of laparoscopic bile duct injuries. Analysis of 252 cases from human factors and cognitive psychology perspective. Ann Surg 2003; 237: 460-9.

16. Strasberg SM. Biliary injury in laparoscopic surgery: part 1. Processes used in determination of standard of care in misidentification injuries. J Am Coll Surg 2005; 201: 598-603.

17. Kozicki I, Durowicz S, Tarnowski W. Czynniki sprzyjające urazom jatrogennym dróg żółciowych podczas cholecystektomii laparoskopowej i sposoby w ich zapobieganiu (Factors favoring iatrogenic injuries of bile ducts during laparoscopic cholecystectomy and methods of their prevention). Post Nauk Med 2011; 24: 42-8.

18. Thompson CM, Saad NE, Quazi RR, et al. Management of iatrogenic bile duct injuries: role of the interventional radiologist. Radiographics 2013; 33: 117-34.

19. Kapoor VK. Hepatectomy for bile duct injury - when repair is not enough! Clin Surg 2016; 1: 1203.

20. Mc Mahon AJ, Fullarton G, Baxter JN, O’Dwyer PJ. Bile duct injury and bile leakage in laparoscopic cholecystectomy. Br J Surg 1995; 82: 307-13.

21. Stewart L, Way LW. Bile duct injuries during laparoscopic cholecystectomy (factors that influence the results of treatment). Arch Surg 1995; 130: 1123-9.

22. Lillemoe KD. Evaluation of suspected bile duct injuries. Surg Endosc 2006; 20: 1638-43.

23. Callery MP. Avoiding biliary injury during laparoscopic cholecystectomy: technical considerations. Surg Endosc 2006; 20: 1654-8.

24. Stewart L, Way LM. Laparoscopic bile duct injuries timing of surgical repair does not influence success rate. A multivariate analysis of factors influencing surgical outcomes. HPB (Oxford) 2009; 11: 516-22.

25. Krahenbuhl L, Sclabas G, Wente MN, et al. Incidence, risk factors, and prevention of biliary tract injuries during laparoscopic cholecystectomy in Switzerland. World j Surg 2001; 25: 1325-30.

26. Sowier S, Sowier A, Wiechowska-Kozłowska A, et al. Initial experience with endoscopic ultrasound-guided gallbladder drainage. Videosurgery Miniinv 2019; 14: 195-202.

27. Schmidt SC, Langrehr JM, Hintze RE, Neuhaus P. Long-term results and risk factors influencing outcome of major bile duct injuries following cholecystectomy. Br J Surg 2005; 92: 76-81.

28. Kaman L, Sanyal S, Behera A, et al. Comparison of major bile duct injuries following laparoscopic cholecystectomy and open cholecystectomy. ANZ J Surg 2006; 76: 788-91.

29. Walsh RM, Henderson JM, Vogt DP, Brown N. Long-term outcome of biliary reconstruction for bile duct injuries from laparoscopic cholecystectomies. Surgery 2007; 142: 450-7.

30. Mercado MA, Chan C, Salgado-Nesme N, Lopez-Rosales F. Intrahepatic repair of bile duct injuries. A comparative study. J Gastrointest Surg 2008; 12: 364-8.

31. Winslow ER, Fialkowski EA, Linehan DC, et al. "Sideways": results of repair of biliary injuries using a policy of side-to-side hepatico-jejunostomy. Ann Surg 2009; 249: 426-34.

32. Nuzzo G, Giuliante F, Giovannini I, et al. Advantages of multidisciplinary management of bile duct injuries occurring during cholecystectomy. Am J Surg 2008; 195: 763-9.

33. Tzovaras G, Dervenis C. Vascular injuries in laparoscopic cholecystectomy: an underestimated problem. Dig Surg 2006; 23: 370-4.

34. Sadegh Fazeli M, Kazemeini AR, Jafarian A, et al. Temporary trans-jejunal hepatic duct stenting in Roux-en-y hepaticojejunostomy for reconstruction of iatrogenic bile duct injuries. Trauma Mon 2016; 21: e21115.

35. Suzuki H, Shimura T, Mochhida Y, et al. To stent or not to stent hepaticojejunostomy - analysis of risk factors for postoperative bile leaks and surgical complication. Hepatogastroenterology 2014; 61: 920-6.

36. Marangoni G, Ali A, Faraj W, et al. Clinical features and treatment of sump syndrome following hepaticojejunostomy. Hepatobiliary Pancreat Dis Int 2011; 10: 261-4.

Received: 19.12.2018, accepted: 16.03.2019. 